\title{
Development and Implementation of an in vitro Culture System for Intact Detached
} Grape Berries

Zhanwu Dai $^{1 *}$, Messaoud Meddar ${ }^{1}$, Serge Delrot $^{2}$ and Eric Gomès ${ }^{2}$

${ }^{1}$ INRA, ISVV, UMR 1287 EGFV, Villenave d'Ornon, France; ${ }^{2}$ Univ. de Bordeaux, ISVV, UMR 1287 EGFV, Villenave d'Ornon, France

*For correspondence: zhanwu.dai@bordeaux.inra.fr

[Abstract] Grape composition depends on the metabolites accumulated and synthesized during grape development. It is of paramount importance for grape growers because of its major role in shaping wine quality. Therefore, understanding the regulation mechanisms that control the accumulation of quality-related metabolites in grape is of both scientific and agronomical interests. The composition of grape berry at harvest is under complex regulation and can be affected by many factors (Conde et al., 2007). The study of the effects of these factors on berries still attached to intact plants can be highly challenging because of the large size of the plants, interplant, intercluster and interberry variability; and because it is complicated to precisely control the nutrients and hormones imported by the berries, and the environment. Therefore, in vitro cultured grape berries are a good model system, which better represents berry anatomy structure (skin and flesh) than grape cell suspensions and nevertheless largely reduces the system complexity compared to whole plant (Bravdo et al., 1990; Pérez et al., 2000; Gambetta et al., 2010). To this end, an in vitro culture system of intact detached grape berries has been developed by coupling greenhouse fruiting-cuttings production and in vitro organ culture techniques (Dai et al., 2014). The cultured berries are able to actively absorb and utilize carbon and nitrogen from the culture medium, and exhibit fruit ripening features such as color changing and softening. This in vitro system may serve to investigate the response of berry composition to environmental and nutrient factors.

\section{Materials and Reagents}

1. Grapevine berries from greenhouse-grown fruiting-cuttings of Vitis vinifera L. cV. Cabernet Sauvignon at various developmental stages (e.g. pea size, green berry, veraison, or later stages). The fruiting-cuttings (i.e. only one primary shoot axis with a single cluster per plant) were prepared as described in Mullins and Rajasekaran (1981) and grown in a naturally illuminated and semi-regulated greenhouse (mean seasonal temperature amplitude $20-35^{\circ} \mathrm{C}$ ) with fungicide treatments every two weeks (Figure 1).

Note: Efficient fungicide treatments are essential to maximally exclude pathogen infection to grape berries and minimize the sterilization step for the in vitro culture. For example, we continuously provide sulfur contained in sulfur diffusion hotbox (Nivola 
Sulphur fl $220 \vee 3 \mathrm{~m}$, Figure $1 \mathrm{~B}$ ) in the greenhouse and treated the vines with armicarb $^{\circledR}$ at $5 \mathrm{~g} / \mathrm{L}$ every two weeks.

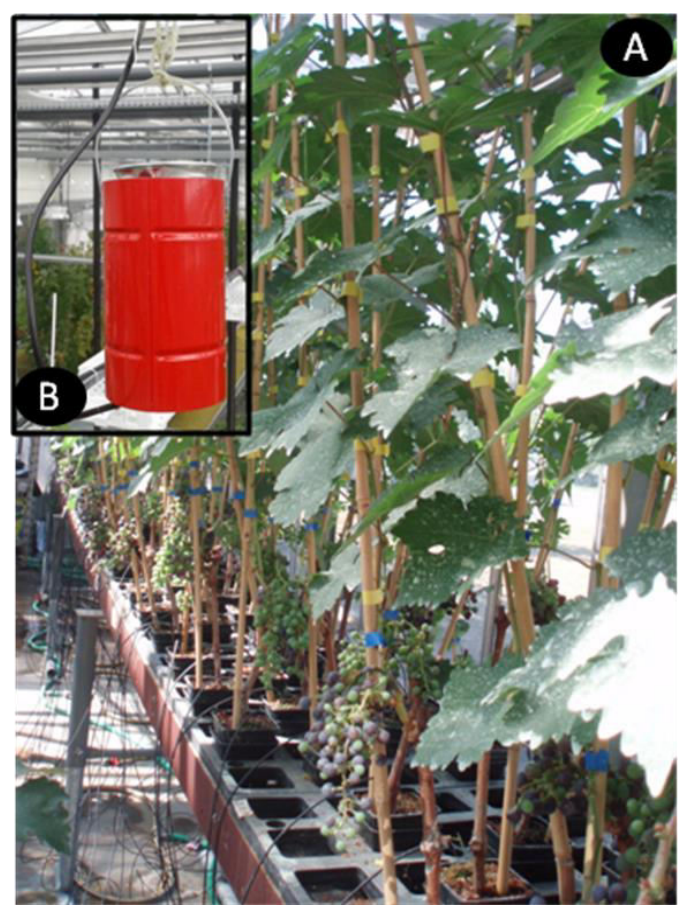

Figure 1. Fruit-bearing cuttings grown in a semi-controlled greenhouse (A) and the sulfur diffusion hotbox (B)

2. $70 \%$ ethanol

3. $\mathrm{NaClO}$ with available chlorine $2 \%$ (Sigma-Aldrich, catalog number: $425044-1 \mathrm{~L}$ )

4. MS (Murashige \& Skoog) medium (Duchefa Biochemie, catalog number: M 0221)

5. Sucrose (Duchefa Biochemie, catalog number: S0809)

6. N-Z-Amine A (Sigma-Aldrich, catalog number: C7290)

7. Vitamines

Myo-inositol $100 \mathrm{mg} / \mathrm{L}$ (Sigma-Aldrich, catalog number: I5125)

Nicotinic acid $1 \mathrm{mg} / \mathrm{L}$ (Sigma-Aldrich, catalog number: N0765)

Pantothenic acid $1 \mathrm{mg} / \mathrm{L}$ (Duchefa Biochemie, catalog number: C0604)

Biotin $0.01 \mathrm{mg} / \mathrm{L}$ (Sigma-Aldrich, catalog number: B4639)

Pyridoxine $\mathrm{HCl} 1 \mathrm{mg} / \mathrm{L}$ (Sigma-Aldrich, catalog number: P9755)

Thiamine $\mathrm{HCl} 1 \mathrm{mg} / \mathrm{L}$ (Sigma-Aldrich, catalog number: T4625)

8. $0.5 \mathrm{M} \mathrm{NaOH}$

9. Agar (Kalys, catalog number: HP 696)

10. EDTA (Sigma-Aldrich, catalog number: E5134)

11. Sterile water

12. MS medium with sucrose and vitamins (see Recipes)

13. $200 x$ vitamin (see Recipes) 


\section{bĭ̈-protocol}

14. 20 mM EDTA (see Recipes)

\section{Equipment}

1. Laminar flow cabinet (Steril-Helios, Figure 2A)

2. Shaker (Dragon lab, sk-330-pro, Figure 2A)

3. Autoclave machine

4. Growth room with constant temperature of $26 \pm 0.5^{\circ} \mathrm{C}$, light period $16 \mathrm{~h} / 8 \mathrm{~h}$ day/night, and light $\sim 50 \mu \mathrm{mol} \mathrm{m} \mathrm{m}^{-2} \mathrm{~s}^{-1}$ (Figure $4 \mathrm{H}$ )

5. Sterilized 6-well plates (Dutscher, catalog number: 353046, Figure 5B)

6. Sterilized plastic boxes with filter (Dutscher, catalog number: E 1650, Figures 3F, 4G, and $5 \mathrm{~A})$

7. Tip plate (Figure $3 \mathrm{~A}$ )

8. Polystyrene (Figure $3 \mathrm{C})$

9. Culture dish (145/20 mm, Greiner Bio-One GmbH, Figure 4E)

10. Sterilization solution container (Figure $2 C-D$ )

11. Colander, forceps, scissors, and blade (Figure 2B)
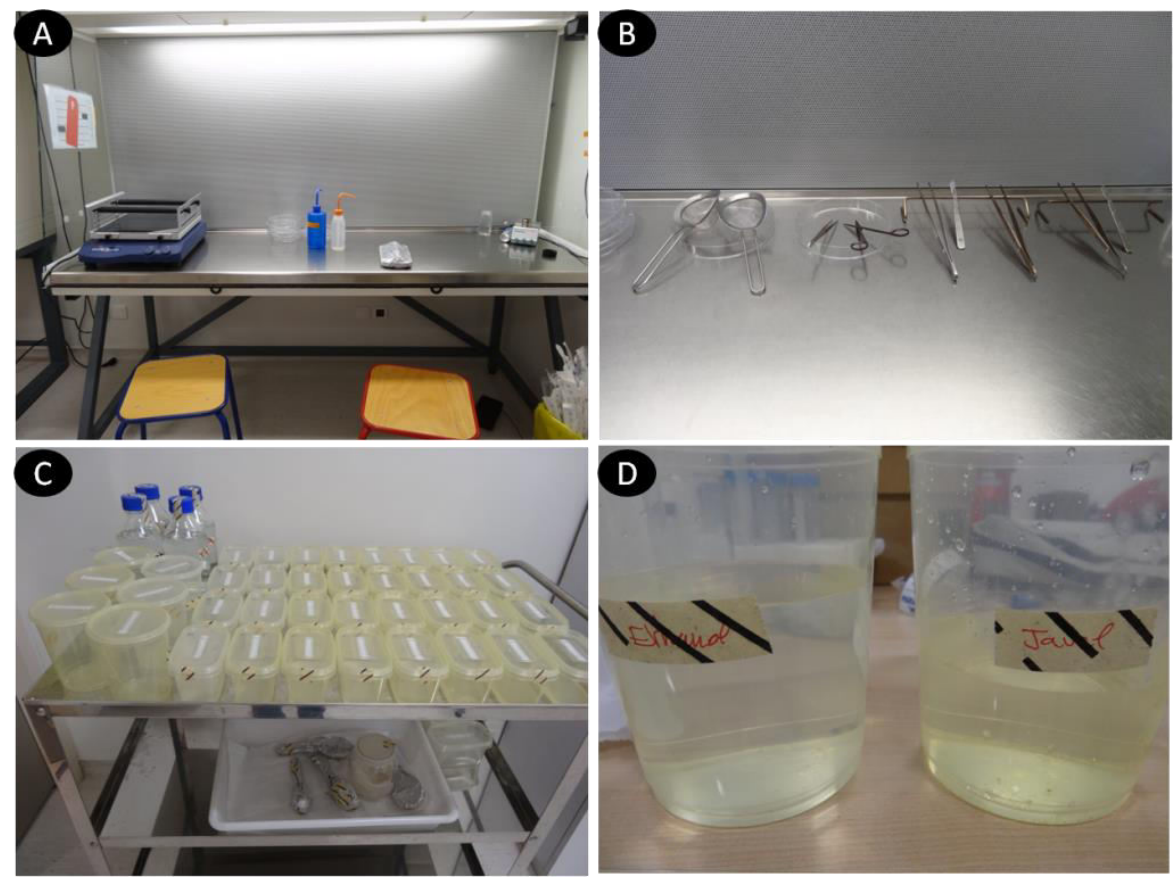

Figure 2. Equipment used in grape berry culture. A. Laminar flow cabinet with a shaker. B. colander, forceps, scissors, and blade. C. solution container, culture boxes, and small materials just after autoclave. D. Sterilized containers for ethanol and $\mathrm{NaClO}$. 


\section{Procedure}

A. Preparation of the floater for liquid medium

1. Collect tip plates and cut them into suitable size for the culture box (Figure $3 A$ and $B$ ).

2. Prepare polystyrene cuboids corresponding to the size of tip plates (Figure $3 C$ ).

3. Put the polystyrene cuboids on the two sides of the tip plates to form a floater (Figure 3D-F).

4. Sterilize floaters with $10 \% \mathrm{NaClO}$ and $90 \%$ ethanol during 20 min each following three rinses with sterile water and then install them in the tissue culture box containing liquid medium.

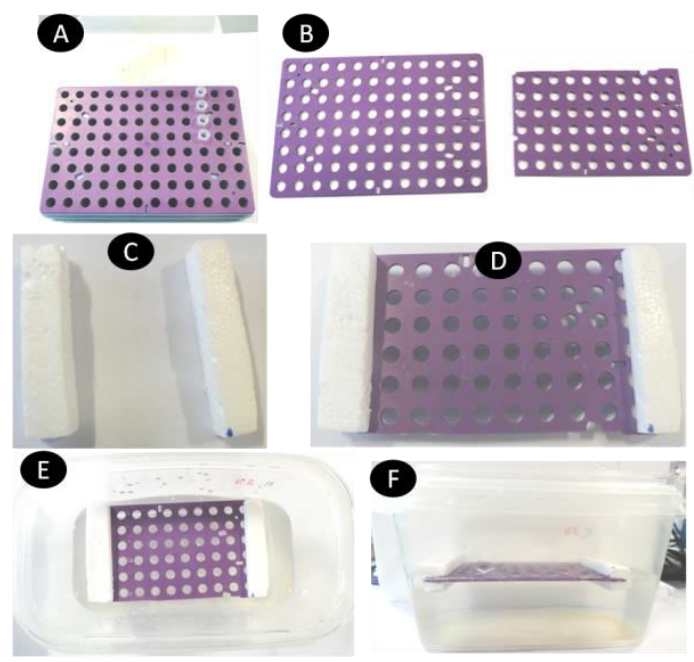

Figure 3. Preparation of the floater for liquid medium. A. Boxes of micropipette tips. B. Cut tip plates to a suitable size for the culture box. C. Prepare two polystyrene cuboids. D. Assemble polystyrene cuboids with tip plate to form the floater. E. Vertical view of the floater inside of a culture box. F. Side view of the floater inside of a culture box.

B. Berry in vitro culture

1. Fruit-bearing cuttings were selected and brought to culture room after removing all leaves (Figure 4A).

2. Grape clusters were excised from the mother plant, and berries were subsequently excised from peduncle with berry pedicel (about $3 \mathrm{~mm}$ ) and dropped immediately into tap water (Figure 4B).

3. Keep tap water running for $15 \mathrm{~min}$ to clean the berries.

4. Put berries into $70 \%$ ethanol for $2 \mathrm{sec}$ and transfer immediately into $\mathrm{NaClO}$ with $2 \%$ available chlorine with a sterilized colander (Figure 4D).

Note: The volume ratio between berry and sterilization solution is important for an efficient sterilization, and a minimum ratio 1:3 (berry/solution) is recommended. 


\section{biö-protocol}

http://www.bio-protocol.org/e1510 Vol 5, Iss 12, Jun 20, 2015

5. Shaking the container of $\mathrm{NaClO}$ with a shaker at 350-450 rpm for 2 min (Figure 4C). Note: Dependent on the status of the greenhouse-grown berries, this step can be extended to 3-5 min. Longer sterilization will hurt berry skin.

6. Transfer berries into sterilized de-ionized water with colander (Figure 4D) and shake at 350-450 rpm for 2 min.

7. Repeat step B6 three times.

8. Transfer fully rinsed berries into $20 \mathrm{mM}$ EDTA solution, which is contained in a big culture dish (Figure 4E).

9. Cut berry pedicel again to about $2 \mathrm{~mm}$ under EDTA solution (Figure 4E), in order to exclude cavitations and to prevent plugging of sieve tubes by callose synthase, a strictly calcium-dependent enzyme.

10. Gently open the culture boxes and quickly put the berries on the solid or liquid culture medium with berry pedicel rooting into culture medium (see Recipes, Figure 4F and Figure 5).

11. Gently put the cover of culture box (Figure $4 \mathrm{G}$ ) and transport all boxes to culture room with constant temperature of $26 \pm 0.5^{\circ} \mathrm{C}$, light period $16 \mathrm{~h} / 8 \mathrm{~h}$ day/night, and light $\sim 50$ $\mu \mathrm{mol} \mathrm{m} \mathrm{s}^{-1}$ (Figure $4 \mathrm{H}$ ).

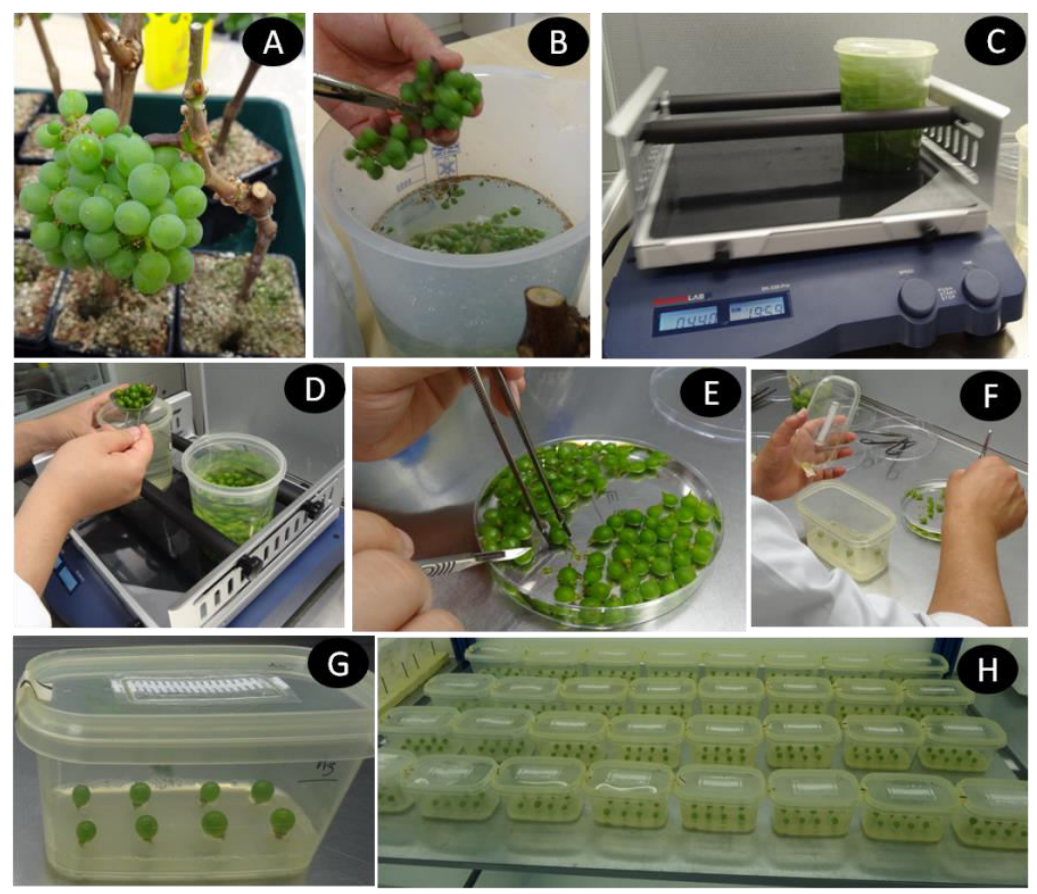

Figure 4. Berry in vitro culture procedure. A. Bring fruit-bearing cuttings to the lab after removing all leaves. B. Excise berries from grape cluster and drop them immediately into tap water. C. Sterilize berries with ethanol and bleach solutions with a shaker. D. Transform the sterilized berries to water for rinsing. E. Recut berry pedicel in $20 \mathrm{mM}$ EDTA. F. Install berries into culture medium. G. Put the cover of tissue culture box. H. Install tissue culture boxes into culture room. 


\section{biö-protocol}

12. Alternative culture methods depending on the desired experiment might be chosen (Figure 5).

13. The berry culture can be maintained as long as 3 months. Since the medium volume is much greater than the berries, there is no need for change of medium. However, it will be very easy to change the medium composition of liquid medium with syringe injection.

Note: Berries are not very tightly fixed on the culture medium, so any movement and transport should be done gently to avoid berry slanting.
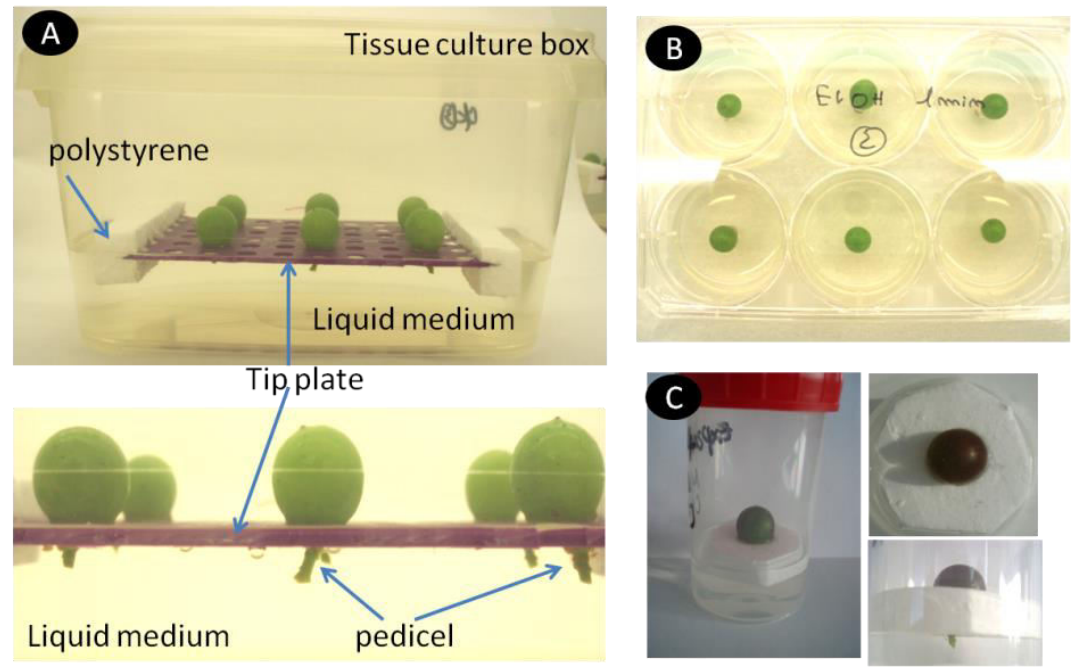

Figure 5. Different types of berry in vitro culture. A. Culture with liquid medium and floater. B. Culture with solid medium in a 6-well plate. C. Culture with liquid medium and floater in a small container.

\section{Recipes}

1. MS medium with sucrose and vitamins

\section{$4.3 \mathrm{~g} \mathrm{M} 0221$}

$20 \mathrm{~g}$ sucrose

\section{$0.25 \mathrm{~g} \mathrm{~N}-Z$-AmineA}

Dissolve in $800 \mathrm{ml}$ deionized water with vortex

$5 \mathrm{ml}$ vitamin 200x (see below)

Adjust $\mathrm{pH}$ to 5.8 with $0.5 \mathrm{M} \mathrm{NaOH}$

Complete to $1 \mathrm{~L}$ with deionized water

Add $9 \mathrm{~g}$ agar for solid medium

Autoclave $\left(120^{\circ} \mathrm{C}, 20 \mathrm{~min}\right)$

For medium containing agar, pour $4 \mathrm{ml}$ medium to each well of the 6-well plate (Figure

$5 \mathrm{~B}$ ); or $50 \mathrm{ml}$ medium to the tissue culture box with special filters allowing gas 
exchange (Figure 4G), before medium concretion and inside of the laminar flow cabinet.

For liquid medium, pour $150 \mathrm{ml}$ medium the tissue culture box with special filters (Figure 5A).

Note: A minimum 2\% of sucrose is needed to prevent berry crack for green Cabernet Sauvignon berries. Different cultivars may have different sensitivity and therefore a suitable sucrose concentration has to be established for other cultivars.

2. $200 x$ vitamin

Dissolve $8 \mathrm{mg}$ biotin into $1 \mathrm{ml}$ de-ionized water with one drop of $\mathrm{KOH}$

$25 \mu \mathrm{l}$ biotin solution

2 g myo-inositol

$20 \mathrm{mg}$ nicotinic acid

$20 \mathrm{mg}$ pantothenic acid

$20 \mathrm{mg}$ pyridoxine $\mathrm{HCl}$

$20 \mathrm{mg}$ thiamine $\mathrm{HCl}$

Dissolve and complete to $100 \mathrm{ml}$ with deionized water

Stored at $-20^{\circ} \mathrm{C}$

3. $20 \mathrm{mM}$ EDTA

Dissolve $7.44 \mathrm{~g}$ EDTA in $1 \mathrm{~L}$ de-ionized water

Autoclave $\left(120^{\circ} \mathrm{C}, 20 \mathrm{~min}\right)$

Stored at room temperature

\section{Acknowledgments}

This work was supported by a specific grant from the Environment and Agronomy division (EA) of the Institut National de la Recherche Agronomique (INRA), France. This protocol was developed based on our previous paper (Dai et al., 2014).

\section{References}

1. Bravdo, B., Shoseyov, O., Ikan, R. and Altman, A. (1990). Monoterpene glycoside biosynthesis in detached grape berries grown in vitro. Physiol Plant 78(1): 93-99.

2. Conde, C., Silva, P., Fontes, N., Dias, A. C. P., Tavares, R. M., Sousa, M. J., Agasse, A., Delrot, S. and Gerós, H. (2007). Biochemical changes throughout grape berry development and fruit and wine quality. Food 1, 1-22.

3. Dai, Z. W., Meddar, M., Renaud, C., Merlin, I., Hilbert, G., Delrot, S. and Gomes, E. (2014). Long-term in vitro culture of grape berries and its application to assess the effects of sugar supply on anthocyanin accumulation. $J$ Exp Bot 65(16): 4665-4677. 
4. Gambetta, G. A., Matthews, M. A., Shaghasi, T. H., McElrone, A. J. and Castellarin, S. D. (2010). Sugar and abscisic acid signaling orthologs are activated at the onset of ripening in grape. Planta 232(1): 219-234.

5. Mullins, M. G., and Rajasekaran, K. (1981). Fruiting cuttings: revised method for producing test plants of grapevine cultivars. Am J Enol Vitic, 32: 35-40.

6. Pérez, F. J., Meza, P., Berti, M. and Pinto, M. (2000). Effect of carbon source and sucrose concentration on growth and hexose accumulation of grape berries cultured in vitro. Plant Cell Tiss Org 61(1): 37-40. 